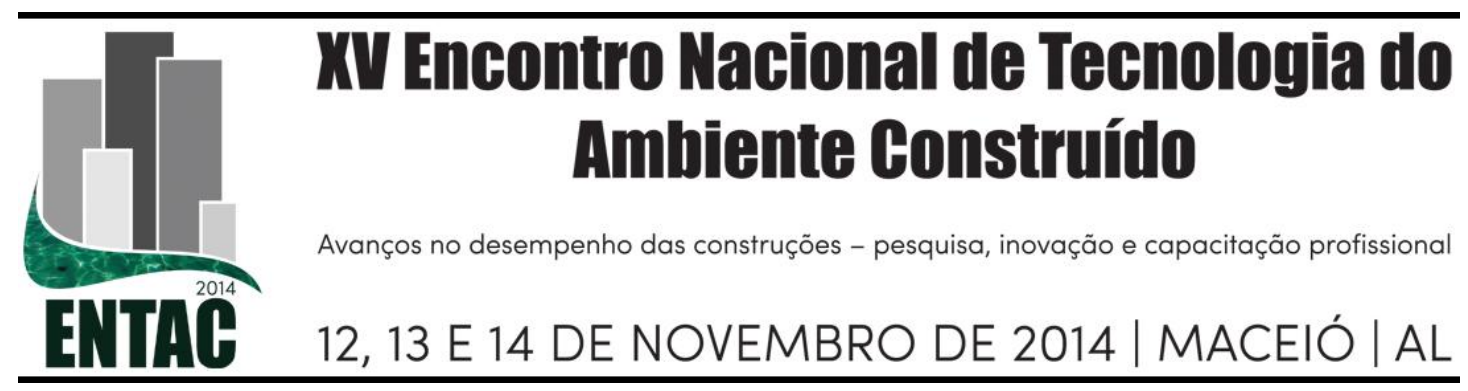

\title{
PROPOSTA DE UMA MODELAGEM DE DADOS PARA AUXILIAR NO CONTROLE INTEGRADO DA PRODUÇÃO E DA QUALIDADE
}

\author{
LEÃO, Cibeli (1); ISATTO, Eduardo (2); FORMOSO, Carlos (3)
}

(1) UFRGS, e-mail: cibeli.leao@gmail.com (2)UFRGS, e-mail: isatto@ufrgs.br, (3) UFRGS, e-mail: formoso@ufrgs.br

\begin{abstract}
RESUMO
A integração entre os controles da produção e da qualidade é considerada como um meio de reduzir a incidência de perdas causadas pela falta de terminalidade das tarefas. No entanto, para facilitar essa integração, é necessário o auxílio da tecnologia de informação, devido à quantidade de dados que devem ser coletados e processados. Assim, o uso de tablets é considerado uma solução para coletar e gerenciar informações no canteiro de obras de uma forma mais eficiente, tendo forte potencial para melhorar o nível de compartilhamento de dados e as práticas de comunicação na indústria da construção e fornecendo oportunidades para inovar os processos de gestão. O objetivo desse artigo é propor uma modelagem de dados para auxiliar no desenvolvimento de aplicações para o controle integrado da produção e da qualidade, e apresentar uma avaliação preliminar da implementação da modelagem proposta em um estudo de caso. O modelo é constituído por três módulos integrados: pacotes de trabalho, qualidade e making-do; sendo possível monitorar a porcentagem de pacotes concluídos, falta de terminalidade das tarefas, ocorrência de pacotes informais, perdas por making-do e indicadores de controle da qualidade. Também são apontadas possíveis integrações entre a modelagem de dados e os modelos BIM.
\end{abstract}

Palavras-chave: Perdas, Controle Integrado, Modelagem de Dados, BIM.

\begin{abstract}
The integration between production and quality control has been pointed out as a means to reduce the incidence of waste caused by unfinished work. However, it is necessary the assistance of information technology to facilitate this integration, due the amount of data that must be collected and processed. Thus, the use of tablets is considered a solution to collect and manage information on construction site in a more efficient manner, with strong potential to improve the data sharing and the communication practices in the construction industry, and providing opportunities to innovate management processes. This paper proposes a data modeling to assist in the development of applications for production and quality control, and it presents the preliminary evaluation of the implementation of the proposed model in a case study. The model consists of three integrated modules: work packages, quality and making-do; and it is possible to monitor the percentage of completed packages, unfinished work, the occurrence of informal packages, making-do waste and indicators of quality control. Also, possible integration between data modeling and BIM models are indicated.
\end{abstract}

Keywords: Waste, Integrated Control, Data Modeling, BIM.

\section{INTRODUÇÃO}

A falta de terminalidade das tarefas é apontada na literatura (ALVES, 2000; SUKSTER, 2005; FIREMAN; FORMOSO; ISATTO, 2013) como um importante problema que 
vem sendo negligenciado pelos sistemas de gestão. Segundo Alves (2000), a terminalidade refere-se à conclusão das tarefas no prazo e com a qualidade determinada, sem a necessidade de retorno posterior de alguma equipe para a realização de retrabalhos ou arremates. A falta de terminalidade das tarefas contribui para o aumento das perdas na construção, devido à necessidade de refazer trabalhos já executados e aumentando a quantidade de trabalho em progresso (ALVES, 2000).

Fireman, Formoso e Isatto (2013) também apontam que a falta de terminalidade contribui para o aumento de pacotes informais, uma vez que os pacotes de retrabalho e arremates, na maioria das vezes, não são planejados durante as reuniões de planejamento semanal, sujeitando-se às incertezas. Ainda, há evidências de que a execução de pacotes informais provoca o aumento das perdas por making-do, que segundo Koskela (2004), ocorrem quando uma tarefa é iniciada sem que todos os itens necessários para sua realização estejam disponíveis. As perdas por making-do são frequentes na construção civil e causam outras perdas como redução da segurança, retrabalho, redução da qualidade e perda de material (SOMMER, 2010; FIREMAN; FORMOSO; ISATTO, 2013).

O sistema Last Planner de controle da produção, proposto por Ballard (2000), foi desenvolvido com o objetivo de melhorar a confiabilidade dos fluxos de trabalho, protegendo a produção dos efeitos da incerteza e reduzindo a variabilidade. Segundo Koskela (2004), o Last Planner utiliza a eliminação das perdas por making-do como um condutor para reduzir a variabilidade e melhorar o sistema de produção como um todo. No entanto, Marosszeky et al. (2002) salientam que, embora a utilização do Last Planner resulte em uma maior transparência e confiabilidade, auxiliando na identificação de alguns obstáculos para atingir uma produção livre de defeitos, a qualidade do produto não é considerada explicitamente nesse sistema.

A integração entre os controles da produção e da qualidade é considerada como um meio de reduzir a incidência de pacotes informais e perdas por making-do. Assim, é proposto por Leão (2014) um modelo de controle integrado da produção e da qualidade, vinculado ao sistema Last Planner, capaz de monitorar perdas na construção civil causadas pela falta de terminalidade e pela execução de pacotes informais. No entanto, para facilitar essa integração, é necessário o auxílio da tecnologia de informação, devido à quantidade de dados que devem ser coletados e processados.

O uso de dispositivos móveis no canteiro de obras, como tablets, tem sido apontado na literatura como uma solução para coletar e gerenciar informações referentes à construção de uma forma mais eficiente (KIM; LIM; KIM, 2011). No entanto, o uso de sistemas informatizados depende em grande parte da formalização de uma ontologia, que, segundo Park et al. (2012), representa o conhecimento como um conjunto de conceitos e suas inter-relações. No caso do uso de dispositivos móveis, abordado nesse artigo, tais ontologias necessitam ser traduzidas em modelos de dados, os quais servem para armazenar as informações relevantes no domínio da aplicação, nesse caso, o controle integrado da produção e da qualidade. Sendo assim, o objetivo desse artigo é propor uma modelagem de dados para auxiliar no desenvolvimento de aplicações para o controle integrado da produção e da qualidade.

Este artigo aborda especificamente obras de empreendimentos horizontais e de natureza repetitiva, apresentando as informações obtidas com a implementação da modelagem proposta em um estudo de caso, e a avaliação preliminar da sua contribuição para os sistemas de gestão. 


\section{REVISÃO DE LITERATURA}

\subsection{Pacotes de Trabalho}

O Sistema Last Planner é um conjunto de ferramentas utilizado para facilitar a implementação de um controle mais efetivo, tendo duas funções principais: o controle da unidade de produção e o controle de fluxo do trabalho (BALLARD, 2000). A primeira função visa fazer melhores atribuições para os trabalhadores através da aprendizagem contínua e de ações corretivas. Já a segunda função está voltada em fazer com que o fluxo de trabalho siga através da unidade de produção na melhor sequência possível (BALLARD, 2000).

O Last Planner pode ser entendido como um mecanismo para transformar o que deveria ser feito em algo que pode ser feito (BALLARD, 2000). Para isso, é realizado o planejamento de médio prazo, no qual é criado um estoque de atividades aptas a serem executadas, a partir do qual é formado o plano de trabalho semanal, ou plano de curto prazo. O plano de curto prazo é um compromisso do último planejador com aquilo que realmente será feito (BALLARD, 2000). Assim, segundo o autor, os pacotes de trabalho planejados devem ser suficientemente detalhados, a fim de permitir que os mesmos sejam preparados e que sua conclusão possa ser identificada claramente. No entanto, Choo e Tommelein (2001) mencionam que um pacote de trabalho não pode ser excessivamente detalhado, a fim de permitir certa flexibilidade aos trabalhadores durante a execução do trabalho.

Choo, Tommelein e Ballard (1999) definem pacote de trabalho como uma determinada quantidade de trabalho similar, ou um conjunto de tarefas, que será feito frequentemente em uma área bem definida, utilizando informações específicas de projeto, material, mão-de-obra e equipamento, e com todos os pré-requisitos completos. Marchesan (2001) ainda sugere que o pacote de trabalho seja definido por uma ação, um elemento e um local.

\subsection{Controle Integrado da Produção e da Qualidade}

Segundo Marosszeky et al. (2002), o controle da qualidade deveria ser realizado no tempo mais próximo possível ao da execução do trabalho, identificando os problemas de qualidade e agindo de forma a limitar sua recorrência. No entanto, os autores afirmam que, geralmente, o controle da qualidade é realizado muito tempo depois da execução da tarefa. Assim, quando os serviços são verificados, os trabalhadores envolvidos na execução já foram para outras áreas do canteiro ou já deixaram a obra por completo. Isso faz com que o tempo entre a detecção do problema e sua correção seja muito longo (MAROSSZEKY et al., 2002).

De acordo com Marosszeky et al. (2002), o controle da qualidade deveria envolver a análise, a inovação, a resolução de problemas e a aprendizagem. Para os autores, o foco do controle da qualidade deveria ser identificar os defeitos em um dia e evitar sua ocorrência no outro. Ainda, quando o controle da qualidade é realizado na origem, a tomada de decisão é mais rápida e ágil, as organizações tornam-se menos burocráticas e mais dinâmicas e a melhoria da qualidade torna-se uma consequência (MAROSSZEKY et al., 2002).

Para Misfeldt e Bonke (2004), a qualidade deve ser controlada e aprovada antes de uma atividade ser dada como concluída, a fim de garantir que as tarefas subsequentes não serão executadas sobre partes da construção que apresentam defeitos. Sukster (2005) ainda ressalta que a utilização de procedimentos e planilhas que integrem os sistemas de controle da produção e da qualidade podem beneficiar ambos os sistemas, 
principalmente em relação ao comprometimento das equipes e à redução das não conformidades. Segundo Arentsen, Tiemersma e Kals (1996), a integração dos controles da produção e da qualidade é considerada como uma solução para garantir o cumprimento do prazo de conclusão e o uso eficiente dos recursos.

\section{MÉTODO DE PESQUISA}

A estratégia de pesquisa adotada para esse trabalho é a pesquisa construtiva, ou design science research, que é uma forma de produção de conhecimento, que segundo Lukka (2003), é usada para produzir construções inovadoras com o objetivo de solucionar problemas do mundo real e fazer uma contribuição para a teoria da disciplina na qual está sendo aplicada.

Como parte de uma pesquisa de mestrado, está sendo desenvolvida uma proposta de modelagem de dados para auxiliar no controle integrado da produção e da qualidade com o uso de tablets. Para o desenvolvimento da modelagem foi utilizada a técnica ERD (Entity Relationship Diagram), sendo que, para a identificação das entidades e suas relações, foram consideradas todas as tabelas necessárias para o controle integrado referente a pacotes de trabalho, perdas por making-do e verificação da qualidade.

A modelagem foi testada na obra de dois empreendimentos horizontais similares de uma empresa construtora de Porto Alegre/RS, financiados pelo Programa Minha Casa Minha Vida. O sistema de controle integrado foi concebido para operar sem a necessidade de rede, através de uma base de dados residente em um desktop (um servidor de BD) e uma base de dados no dispositivo móvel (para a coleta de dados). Para testar a modelagem proposta, foram inicialmente criados bancos de dados nas plataformas MySQL (para o servidor BD desktop) e Sqlite (para o dispositivo móvel). Tais bancos de dados possuíam essencialmente a mesma estrutura (modelo de dados). A base de dados do dispositivo móvel seria atualizada no início da semana, a partir dos pacotes de trabalho programados para a semana na reunião de planejamento de curto prazo, sendo que, ao final da semana, os dados coletados no período seriam transferidos para a base de dados do servidor. Segue-se então um processo de análise crítica dos dados e sua consolidação, para dar-se início a um novo ciclo de planejamento e controle semanal.

Para o teste da modelagem de dados optou-se pelo uso de planilhas eletrônicas e arquivos no formato $M S$ Excel tanto para o armazenamento das tabelas como para a interface para coleta de dados, ambos em um tablet com sistema operacional Android e tela de 7". Para a interface de coleta de dados era necessário um aplicativo de visualização e edição de planilhas que possibilitasse o uso da função PROCV (procura vertical) e do recurso validação de dados, disponíveis em softwares como o MS Excel e LibreOffice. Foram avaliados seis aplicativos para a plataforma Android, dos quais foi selecionado o Kingsoft Office, pois além de possibilitar o uso das funções e recursos necessários, o aplicativo era gratuito.

Em um primeiro momento, o teste foi realizado pela própria pesquisadora para identificação de melhorias, e após alguns refinamentos, a modelagem foi utilizada por outros pesquisadores e também por usuários da empresa, a fim de validar sua aplicabilidade. 


\section{RESULTADOS}

\subsection{Proposta de modelagem de dados}

Toda modelagem implica em algum tipo de propósito ou intenção. A ontologia aqui descrita busca o atendimento simultâneo e de forma integrada de três objetivos distintos: o controle da qualidade, o controle da produção e a identificação dos eventos de making-do, visando um planejamento mais eficaz e a redução de perdas. A cada um destes objetivos corresponde um módulo específico no modelo de dados.

O modelo de dados foi construído utilizando-se o software MySQLWorkbench, para a plataforma $M y S Q L$, sendo este posteriormente portado para as plataformas Sqlite e tabelas de planilhas eletrônicas. Este modelo foi mantido em sincronia em todas essas plataformas, na medida em que os dados coletados a partir dos dispositivos móveis demandavam refinamentos no modelo inicialmente proposto. $\mathrm{O}$ modelo, em sua configuração final, encontra-se representado no diagrama ERD da Figura 1.

\section{Figura 1 - Diagrama ERD}

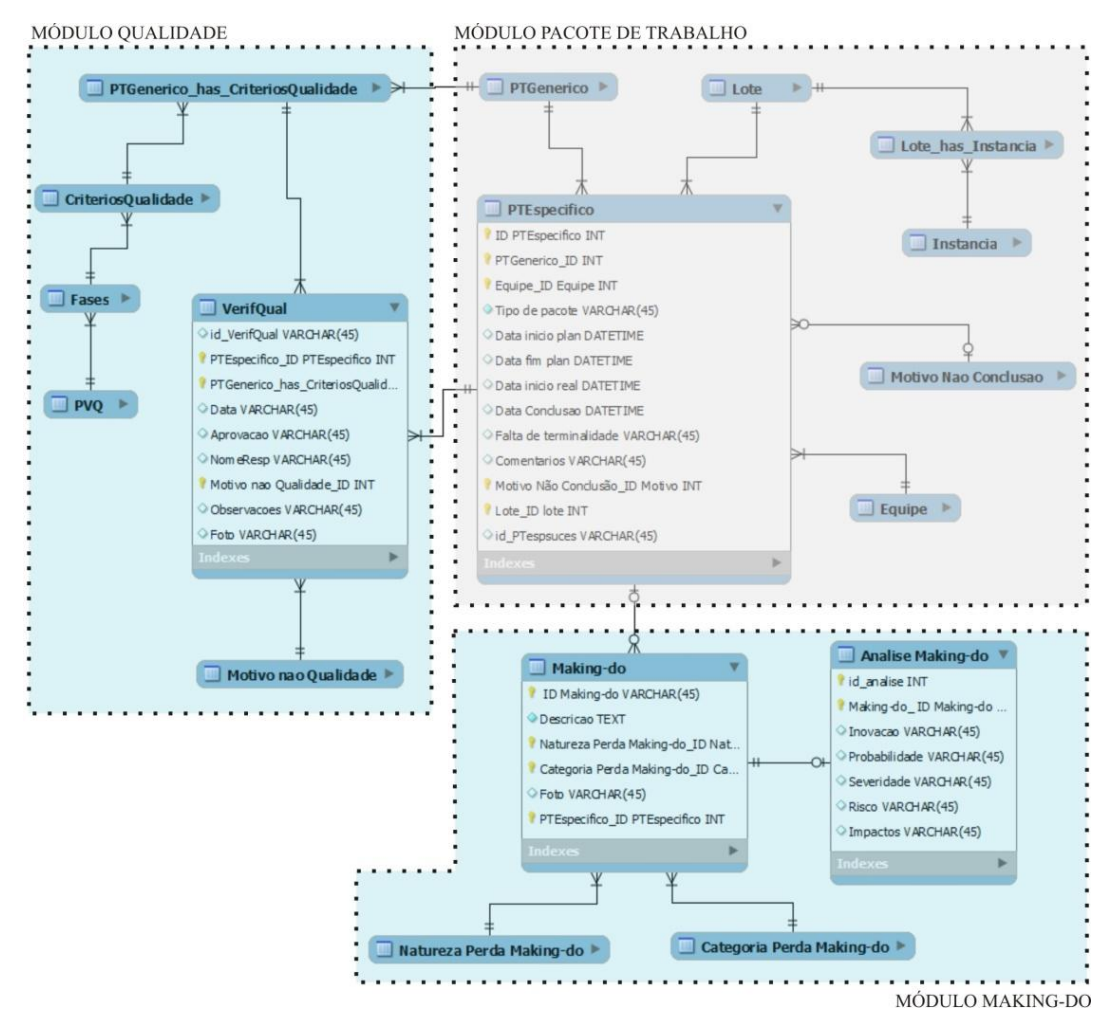

\subsubsection{Módulo pacote de trabalho}

O pacote de trabalho é a unidade de controle do modelo proposto. Denominada como PTEspecifico no modelo, ele deve corresponder a uma quantidade bem definida de serviço, a ser executado por um operário e equipe, e que segundo Marchesan (2001), deve ser representada por uma ação em um determinado elemento e um determinado local. Neste caso, a ação representa um tipo de trabalho ou serviço a ser realizado (p.ex., realizar a concretagem) e o elemento representa uma parte bem definida da edificação (p.ex., paredes). Como as obras repetitivas se caracterizam pela existência de uma ou mais unidades de repetição (como por exemplo, os vários modelos de casas), é natural que os pacotes de trabalho realizados em cada uma das casas também sigam um padrão de repetição, definido pelas possíveis combinações "ação + elemento" associadas ao 
modelo de casa. Tal combinação foi aqui considerada como um "pacote de trabalho genérico" (PTGenerico). Assim, o pacote de trabalho (PTEspecifico) pode ser visto como uma associação entre um pacote de trabalho genérico e um local, este representado por uma ou mais instâncias da unidade-base (neste caso, as diferentes casas). Na ontologia proposta, Instancia representa cada uma das casas, Lote representa uma quantidade de casas executadas simultaneamente em um mesmo pacote de trabalho, e Lote_has_instancia representa a composição de cada lote específico em termos das casas que o compõe.

Quando um pacote de trabalho é atribuído a uma equipe ou operário, este é associado a um recurso existente (Equipe) e uma programação (datas planejadas de início e fim). Durante o controle da produção, a data de início dos pacotes de trabalho é registrada à medida que os mesmos são iniciados, o mesmo ocorrendo em relação a sua conclusão. Todos estes atributos (equipe, datas de início e fim planejados, datas de início e fim observadas) são partes integrantes de um pacote de trabalho, sendo definidos ao longo da sua existência.

A empresa estudada tem por regra associar quaisquer atrasos na execução de pacotes com um tipo de causa, favorecendo a posterior análise e estratificação das causas mais significativas de atrasos. Assim, quando um pacote de trabalho não é concluído dentro da semana prevista, deve ser registrado o Motivo_não_Conclusão, selecionando um dos motivos pré-definidos nessa tabela.

Diferentes tipos de pacotes de trabalho foram identificados ao longo do estudo. Um pacote de trabalho idealmente é concebido durante o planejamento da execução, na reunião de planejamento de curto prazo. Neste caso, é denominado como um pacote "formal". Porém, durante o controle da produção podem eventualmente ocorrer situações onde trabalhadores ou equipes estejam realizando atividades não planejadas. Neste caso, optou-se por considerá-las também como pacotes de trabalho, porém do tipo "informal”, ou seja, cuja realização não foi previamente planejada.

Confirmando as situações mencionadas por Sukster (2005), Righi e Isatto (2011) e Fireman, Formoso e Isatto (2013), diversos casos foram observados nos quais um pacote de trabalho anteriormente dado como concluído pelas equipes e pela engenharia era posteriormente considerado inconcluso, ou por falta de terminalidade ou por não atender aos padrões de qualidade exigidos. Por exemplo, ocorreu de alguns pacotes de trabalho relacionados à regularização ou à textura da parede, serem dados como concluído pela equipe, mas faltava regularizar ou texturizar alguma parte da parede, como a verga das portas ou janelas. Em tais casos, para se evitar a execução da conclusão ou reparo como um pacote informal, é criado um novo pacote idêntico ao anterior, do tipo "retrabalho". Este terceiro tipo serve como um sinalizador para informar a falta de terminalidade do pacote de trabalho original, para que sua conclusão seja planejada e assim evitar sua execução como um pacote informal.

\subsubsection{Módulo making-do}

O módulo making-do tem como principal objetivo identificar as situações sub-ótimas de produção, caracterizadas pela decisão de se executar um pacote de trabalho sem que as pré-condições para sua realização estejam plenamente atendidas. Ele é composto pelas entidades Making-do, Categoria_Perda_Making-do, Natureza_Perda_Making-do e Análise_Making-do.

Uma ocorrência de making-do (entidade Making-do) consiste basicamente numa improvisação identificada durante a execução dos pacotes de trabalho (PTEspecifico). Desta forma, toda ocorrência de making-do deve necessariamente estar associada com 
um pacote de trabalho específico onde o evento ocorreu. A identificação das perdas por making-do é realizada conforme o método proposto por Sommer (2010).

Cada ocorrência de making-do deve ser associada a uma categoria de perda e ao tipo de pré-requisito ausente. A entidade Categoria_Perda_Making-do diz respeito às categorias pré-definidas no sistema, definidas por Sommer (2010) e Fireman, Formoso e Isatto (2013), que são acesso/mobilidade, ajustes de componentes, área de trabalho, armazenamento, equipamento/ferramentas, instalações provisórias, proteção e sequenciamento. Sempre que uma nova categoria de perda é identificada, a mesma deve ser cadastrada nessa tabela. Na entidade Natureza_Perda_Making-do são listados os pré-requisitos, apontados por Sommer (2010) como sendo: informação, materiais e componentes, mão-de-obra, equipamentos/ferramentas, espaço, serviços interligados, condições externas e instalações. Novos pré-requisitos identificados devem ser adicionados a esta lista.

Toda ocorrência de making-do deve posteriormente ser analisada, de forma a evitar sua recorrência. Na entidade Análise_Making-do pode ser realizada a avaliação das improvisações identificadas durante a realização dos pacotes de trabalho. Nessa tabela constam se a improvisação identificada trata-se de uma inovação para o sistema produtivo, a probabilidade de sua ocorrência e sua severidade, assim como o risco e os impactos gerados na produção.

\subsubsection{Módulo de controle da qualidade}

O módulo de controle da qualidade é parte integrante do Sistema da Qualidade da empresa, e deve armazenar todos os registros de qualidade do processo produtivo, servindo não apenas para identificar os problemas de qualidade e saneá-los, mas também para dar condições de rastreabilidade de causas prováveis no caso de futuros problemas de qualidade. As entidades consideradas para o controle da qualidade neste artigo (PVQ, Fase, Criterios_Qualidade, PTGenerico_has_Criterio, Verificação_Qualidade e Motivo_não_Qualidade) foram baseadas no referido sistema da qualidade, e são descritas a seguir.

Uma Planilha de Verificação da Qualidade (PVQ) corresponde a um determinado serviço ou processo, a qual é parte integrante da documentação do sistema de gestão da qualidade da empresa. Cada um dos itens a serem avaliados corresponde a um critério de qualidade (entidade Criterios_Qualidade), os quais podem possuir especificações e tolerâncias a eles associados. Tais critérios podem ser agrupados em termos das fases do serviço (entidade Fase) em que ocorrem, as quais são dependentes do tipo de serviço que dizem respeito e encontram-se pré-definidas no sistema da qualidade da empresa. Por exemplo, a PVQ de execução das paredes de concreto possui itens a serem avaliados para cada uma das seguintes fases do serviço, as quais são específicas para esse serviço: início do serviço, marcação, armadura, instalações elétricas, instalações hidráulicas, instalação de gás, montagem da fôrma, travamento e escoramento da fôrma, montagem de andaimes, antes e durante a concretagem, e pós-desforma.

Cada pacote de trabalho genérico pode ter vários critérios de qualidade a serem avaliados, assim como um critério de qualidade pode estar relacionado a mais de um pacote genérico. Por isto, existe uma entidade de relacionamento denominada PTGenerico_has_Criterio a qual exprime tais relações.

A cada uma destas relações podem corresponder uma ou mais verificações da qualidade (entidade Verificação_Qualidade), que consistem basicamente na verificação da qualidade dos pacotes específicos executados em relação a cada um destes critérios. Assim, tais verificações da qualidade correspondem aos registros de cada uma das 
várias inspeções de qualidade realizadas, constituindo-se na entidade central do módulo qualidade. Quando um pacote de trabalho não é aprovado na verificação da qualidade, deve ser identificada a causa que originou a falta de qualidade, conforme apontado por Fireman, Formoso e Isatto (2013), os quais podem ser: tarefa precedente concluída sem qualidade, material inapropriado, negligência da força de trabalho, baixa instrução da força de trabalho e pré-requisito não disponível. Esses motivos pré-definidos são cadastrados na entidade Motivo_não_Qualidade, a qual pode eventualmente ser ampliada para incluir outros motivos.

\subsubsection{Interface para coleta de dados}

A interface para coleta de dados utilizada para testar a modelagem de dados é composta por três planilhas (Figura 2): pacotes, making-do e qualidade. Na planilha pacotes constam os pacotes de trabalho específicos planejados para a semana e também são inseridos os pacotes informais identificados no canteiro de obras. A função dessa planilha é controlar a execução dos pacotes de trabalho, registrando as datas de início e de conclusão. Na planilha "Making-do" são registradas as improvisações identificadas durante a execução dos pacotes de trabalho, sendo classificadas quanto à categoria e natureza. Já a planilha qualidade contém os critérios de qualidade que devem ser verificados em cada pacote de trabalho específico. Assim que o pacote específico é concluído, deve-se verificar sua conformidade com os critérios pré-estabelecidos nessa planilha, registrando-se a data de verificação da qualidade, se o pacote foi aprovado (S/N) e o nome do responsável pela verificação.

\section{Figura 2 - Interface para coleta de dados}

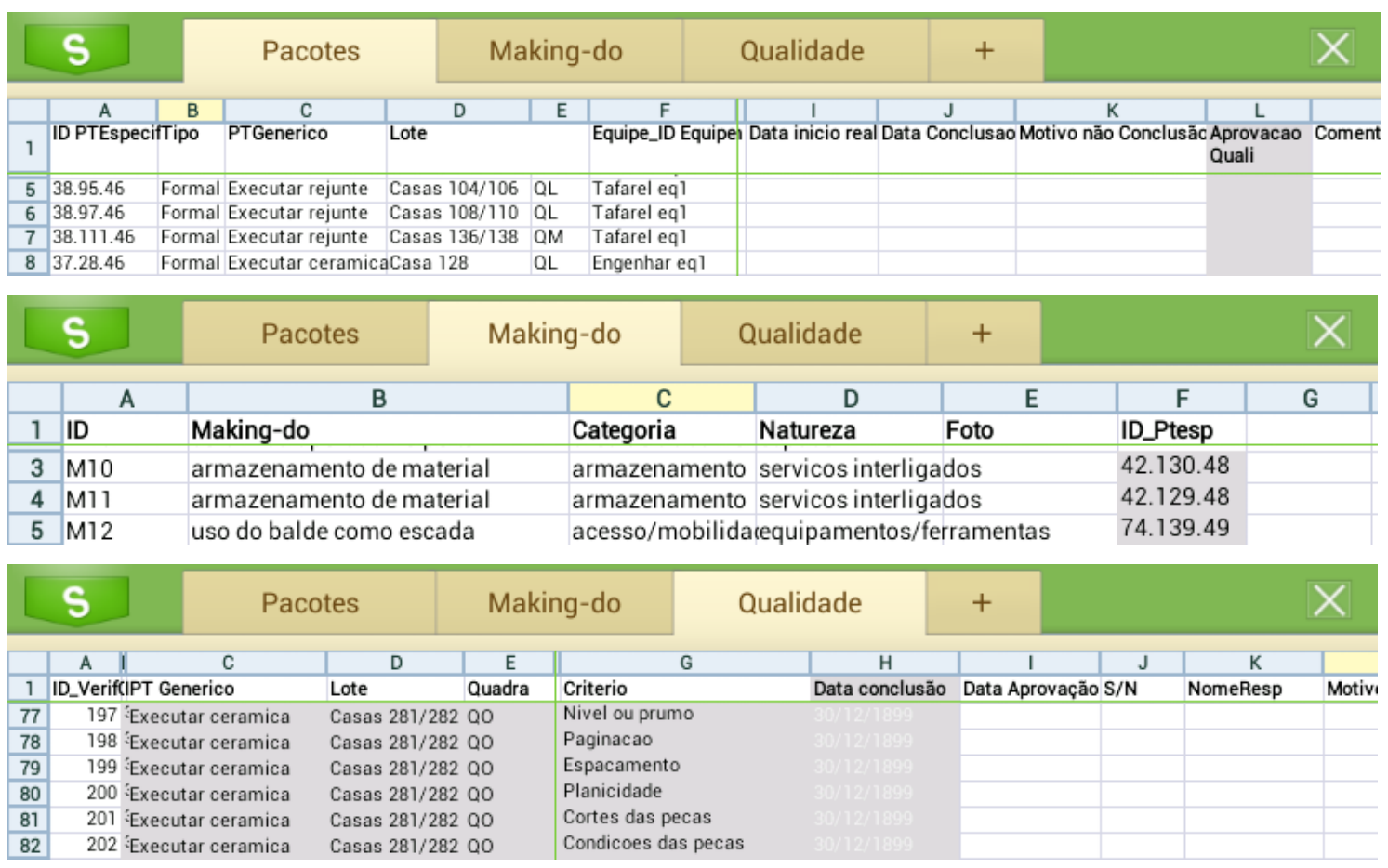

\subsection{Resultados da aplicação da modelagem proposta}

A modelagem de dados proposta permitiu que fossem coletados e processados dados relacionados à conclusão de pacotes de trabalho, execução de pacotes informais, falta de terminalidade das atividades, perdas por making-do e verificação da qualidade, sendo possível calcular indicadores como PPC (porcentagem de pacotes concluídos), PPCQ 
(porcentagem de pacotes concluídos com qualidade), PPFT (porcentagem de pacotes com falta de terminalidade) e PPCR (porcentagem real de pacotes concluídos). Uma discussão mais aprofundada sobre esses dados pode ser encontrada em Leão (2014).

A utilização do conceito de pacotes genéricos permitiu que cada pacote de trabalho fosse relacionado com critérios de qualidade pré-definidos, facilitando a verificação da qualidade assim que os pacotes eram concluídos. Além disso, o uso de tablets para a coleta de dados foi importante para reduzir a quantidade de papéis que deve ser utilizada no canteiro de obras para realizar o controle, e possibilitou que a informação fosse disponibilizada mais facilmente, evitando ter que reescrever os dados.

Ao avaliar a modelagem de dados proposta, os usuários apontaram a possibilidade em manter um histórico da obra no banco de dados e a melhor rastreabilidade das informações. $\mathrm{O}$ armazenamento das informações no banco de dados também facilitou a análise dos problemas mais recorrentes, o que possibilita a tomada de ações preventivas, auxiliando nos ciclos de aprendizagem do sistema Last Planner.

Ainda, foi reportada pelos usuários a dificuldade em visualizar os dados coletados no modelo de controle integrado. Assim, são sugeridas duas possíveis formas de integração do sistema de controle com modelos BIM. A primeira consiste na possibilidade de visualizar os dados referentes aos pacotes de trabalho específicos diretamente no modelo BIM. Neste caso deve haver uma ligação entre os pacotes genéricos (PTGenerico) e os vários elementos que compõem o modelo. A outra possibilidade identificada surgiu da dificuldade em localizar as instâncias (no caso, as casas) no canteiro de obras, devido à sua extensão. Neste caso, poderia haver uma associação da entidade instância com um modelo de implantação do empreendimento, inclusive com a possibilidade de emprego de um sistema georreferencial. No entanto, tais aplicações não foram incluídas no escopo da presente pesquisa.

\section{CONSIDERAÇÕES FINAIS}

A integração entre os controles da produção e da qualidade é considerada como um meio de reduzir a incidência de pacotes informais e perdas por making-do. No entanto, para facilitar essa integração, é necessário o auxílio da tecnologia de informação, devido à quantidade de dados que devem ser coletados e processados. Assim, foi desenvolvida uma modelagem de dados para dar suporte ao controle integrado da produção e da qualidade.

A modelagem de dados proposta é composta por três módulos integrados (pacote de trabalho, making-do e qualidade), permitindo que sejam coletados e processados dados relacionados à conclusão de pacotes de trabalho, execução de pacotes informais, falta de terminalidade das atividades, perdas por making-do e verificação da qualidade.

A modelagem foi avaliada a partir da sua aplicação na obra de um empreendimento horizontal de baixa renda, sendo apontados pelos usuários os benefícios em utilizar o banco de dados para a integração dos controles da produção e da qualidade. Também foram identificados benefícios que poderiam resultar da utilização do modelo proposto associado à tecnologia BIM.

\section{REFERÊNCIAS}

ALVES, T. Diretrizes para a gestão dos fluxos físicos em canteiros de obras: proposta baseada em estudos de caso. Dissertação (Mestrado em Engenharia) - Programa de PósGraduação em Engenharia Civil, Universidade Federal do Rio Grande do Sul, Porto Alegre, 2000. 
ARENTSEN, A. L.; TIEMERSMA, J. J.; KALS, H. J. J. The integration of quality control and shop floor control. International Journal of Computer Integrated Manufacturing, v. 9, n. 2, p. 113-130, jan. 1996.

BALLARD, H. The last planner system of production control. Thesis (Ph.D) - School of Civil Engineering, Faculty of Engineering, University of Birmingham, Birmingham, 2000.

CHOO, H.; TOMMELEIN, I. Requirements and barriers to adoption of last planner computer tools. In: Annual Conference of the International Group for Lean Construction, 9, 2001. Proceedings...Singapura: IGLC, 2001.

CHOO, H.; TOMMELEIN, I.; BALLARD, G. WorkPlan: Constraint-based database for work package scheduling. Journal of Construction Enginnering and Management, v. 125, n. 3, p. 151-160, 1999.

FIREMAN, M. C. T.; FORMOSO, C. T.; ISATTO, E. L. Integrating production and quality control: monitoring making-do and unfinished work. In: Annual Conference of the International Group for Lean Construction, 21, 2013. Proceedings...Fortaleza: IGLC, 2013.

KIM, C.; LIM, H.; KIM, H. Mobile computing platform for construction site management. In: International Symposium on Automation and Robotics in Construction, 28, Seoul, Korea. Proceedings...2011.

KOSKELA, L. Making-do - the eighth category of waste. In: Annual Conference of the International Group for Lean Construction, 12, 2004. Proceedings...Dinamarca: IGLC, 2004.

LEÃO, C.F. Proposta de modelo para controle integrado da produção e da qualidade utilizando tecnologia de informação. Dissertação (Mestrado em Engenharia) - Programa de Pós-Graduação em Engenharia Civil, Universidade Federal do Rio Grande do Sul, Porto Alegre, 2014.

LUKKA, K. The constructive research approach. In: Ojala, L. \& Hilmola, O-P. (eds.) Case study research in logistics. Publications of the Turku School of Economics and Business Administration, Series B1, p. 83-101, 2003.

MARCHESAN, P. R. C. Modelo integrado de gestão de custos e controle da produção para obras civis. Dissertação (Mestrado em Engenharia) - Programa de Pós-Graduação em Engenharia Civil, Universidade Federal do Rio Grande do Sul, Porto Alegre, 2001.

MAROSSZEKY, M. et al. Quality management tools for lean production: moving from enforcement to empowerment. In: Annual Conference of the International Group for Lean Construction, 10, 2002. Proceedings...Gramado: IGLC, 2002.

MISFELDT, E.; BONKE, S. Quality control in lean construction. In: Annual Conference of the International Group for Lean Construction, 12, 2004. Proceedings...Copenhagen: IGLC, 2004.

PARK, C.-S. et al. A Framework for Proactive Construction Defect Management using BIM, Augmented Reality and Ontology-based Data Collection Template. Automation in Construction, n. 33, p. 61-71, out. 2013.

RIGHI, M.; ISATTO, E. L. Sistema de controle da qualidade e planejamento de curto prazo na construção civil: integração e compartilhamento de informações. VII Simpósio Brasileiro de Gestão e Economia da Construçao, Belém, 2011.

SOMMER, L. Contribuições para um método de identificação de perdas por improvisação em canteiros de obras. Dissertação (Mestrado em Engenharia) - Programa de Pós-Graduação em Engenharia Civil, Universidade Federal do Rio Grande do Sul, Porto Alegre, 2010.

SUKSTER, R. A integração entre o sistema de gestão da qualidade e o planejamento e controle da produção em empresas construtoras. Dissertação (Mestrado em Engenharia) Programa de Pós-Graduação em Engenharia Civil, Universidade Federal do Rio Grande do Sul, Porto Alegre, 2005. 